\title{
Thermodynamics of hydrogen bonding in hydrophilic and hydrophobic media
}

\section{David van der Spoel ${ }^{1} \quad$ Paul J. van Maaren ${ }^{1}$ \\ Per Larsson $^{2} \quad$ Nicuşor Tîmneanu ${ }^{1}$}

December 12, 2005

1: Dept. of Cell and Mol. Biology, Uppsala University, Husargatan 3, Box 596,

SE-751 24 Uppsala, Sweden, tel: 46-18-4714205, fax: 46-18-511755, spoel@xray.bmc.uu.se

2: Stockholm Bioinformatics Center, Stockholm University, SE-10691 Stock-

holm, Sweden 


\section{Supplementary material}

Table 1: Kinetics parameters $k$ and $k^{\prime}(1 / \mathrm{ps})$ for different $\mathrm{MeOH}$ concentrations and different temperatures. Numbers are averages over three different simulations, errors within parentheses.

\begin{tabular}{ccccccc}
\hline Mass & \multicolumn{2}{c}{$288.15 \mathrm{~K}$} & \multicolumn{2}{c}{$298.15 \mathrm{~K}$} & \multicolumn{2}{c}{$308.15 \mathrm{~K}$} \\
$\%$ & $k$ & $k^{\prime}$ & $k$ & $k^{\prime}$ & $k$ & $k^{\prime}$ \\
\hline 0 & $0.704(0.001)$ & $2.292(0.004)$ & $0.832(0.001)$ & $2.473(0.004)$ & $0.958(0.001)$ & $2.620(0.002)$ \\
10 & $0.625(0.001)$ & $2.406(0.007)$ & $0.749(0.001)$ & $2.627(0.002)$ & $0.871(0.000)$ & $2.804(0.003)$ \\
20 & $0.561(0.001)$ & $2.517(0.006)$ & $0.680(0.001)$ & $2.775(0.003)$ & $0.798(0.000)$ & $2.976(0.000)$ \\
30 & $0.511(0.002)$ & $2.671(0.010)$ & $0.621(0.001)$ & $2.944(0.008)$ & $0.734(0.001)$ & $3.175(0.006)$ \\
40 & $0.468(0.001)$ & $2.860(0.003)$ & $0.572(0.001)$ & $3.165(0.006)$ & $0.678(0.001)$ & $3.409(0.010)$ \\
50 & $0.435(0.001)$ & $3.115(0.006)$ & $0.532(0.001)$ & $3.448(0.014)$ & $0.632(0.001)$ & $3.695(0.009)$ \\
60 & $0.412(0.002)$ & $3.53(0.03)$ & $0.501(0.001)$ & $3.866(0.013)$ & $0.594(0.002)$ & $4.093(0.011)$ \\
70 & $0.405(0.001)$ & $4.267(0.016)$ & $0.482(0.004)$ & $4.48(0.04)$ & $0.565(0.002)$ & $4.658(0.007)$ \\
80 & $0.410(0.003)$ & $5.26(0.06)$ & $0.479(0.002)$ & $5.38(0.03)$ & $0.554(0.002)$ & $5.46(0.03)$ \\
90 & $0.423(0.001)$ & $6.29(0.04)$ & $0.486(0.001)$ & $6.29(0.03)$ & $0.548(0.001)$ & $6.17(0.02)$ \\
100 & $0.420(0.002)$ & $5.91(0.02)$ & $0.487(0.001)$ & $6.024(0.000)$ & $0.551(0.001)$ & $5.99(0.02)$ \\
\hline
\end{tabular}


Table 2: Kinetics parameters $k$ and $k^{\prime}(1 / \mathrm{ps})$ for different EtOH concentrations and different temperatures. Numbers are averages over three different simulations, errors within parentheses.

\begin{tabular}{ccccccc} 
Mass & \multicolumn{2}{c}{$288.15 \mathrm{~K}$} & \multicolumn{2}{c}{$298.15 \mathrm{~K}$} & \multicolumn{2}{c}{$308.15 \mathrm{~K}$} \\
$\%$ & $k$ & $k^{\prime}$ & $k$ & $k^{\prime}$ & $k$ & $k^{\prime}$ \\
\hline 0 & $0.704(0.001)$ & $2.292(0.004)$ & $0.832(0.001)$ & $2.473(0.004)$ & $0.958(0.001)$ & $2.620(0.002)$ \\
10 & $0.608(0.001)$ & $2.415(0.009)$ & $0.733(0.000)$ & $2.646(0.004)$ & $0.859(0.001)$ & $2.828(0.003)$ \\
20 & $0.529(0.001)$ & $2.490(0.002)$ & $0.649(0.002)$ & $2.765(0.007)$ & $0.772(0.001)$ & $2.979(0.003)$ \\
30 & $0.464(0.002)$ & $2.536(0.008)$ & $0.580(0.005)$ & $2.86(0.03)$ & $0.699(0.002)$ & $3.115(0.006)$ \\
40 & $0.407(0.002)$ & $2.532(0.010)$ & $0.518(0.001)$ & $2.901(0.006)$ & $0.639(0.001)$ & $3.233(0.003)$ \\
50 & $0.367(0.006)$ & $2.62(0.04)$ & $0.471(0.003)$ & $3.009(0.016)$ & $0.580(0.000)$ & $3.348(0.007)$ \\
60 & $0.326(0.002)$ & $2.67(0.03)$ & $0.425(0.003)$ & $3.13(0.03)$ & $0.526(0.001)$ & $3.509(0.007)$ \\
70 & $0.297(0.002)$ & $2.87(0.03)$ & $0.384(0.002)$ & $3.330(0.013)$ & $0.479(0.002)$ & $3.750(0.012)$ \\
80 & $0.277(0.004)$ & $3.37(0.06)$ & $0.353(0.005)$ & $3.83(0.05)$ & $0.440(0.003)$ & $4.26(0.02)$ \\
90 & $0.286(0.004)$ & $4.78(0.09)$ & $0.346(0.002)$ & $5.03(0.04)$ & $0.422(0.005)$ & $5.45(0.10)$ \\
100 & $0.311(0.004)$ & $6.85(0.07)$ & $0.369(0.003)$ & $7.08(0.06)$ & $0.432(0.002)$ & $7.21(0.05)$ \\
\hline
\end{tabular}


Table 3: Kinetics parameters $k$ and $k^{\prime}(1 / \mathrm{ps})$ for different $\mathrm{PrOH}$ concentrations and different temperatures. Numbers are averages over three different simulations, errors within parentheses.

\begin{tabular}{ccccccc} 
Mass & \multicolumn{2}{c}{$288.15 \mathrm{~K}$} & \multicolumn{2}{c}{$298.15 \mathrm{~K}$} & \multicolumn{2}{c}{$308.15 \mathrm{~K}$} \\
$\%$ & $k$ & $k^{\prime}$ & $k$ & $k^{\prime}$ & $k$ & $k^{\prime}$ \\
\hline 0 & $0.704(0.001)$ & $2.292(0.004)$ & $0.832(0.001)$ & $2.473(0.004)$ & $0.958(0.001)$ & $2.620(0.002)$ \\
10 & $0.617(0.001)$ & $2.419(0.002)$ & $0.747(0.000)$ & $2.648(0.002)$ & $0.874(0.000)$ & $2.828(0.003)$ \\
20 & $0.558(0.004)$ & $2.494(0.009)$ & $0.689(0.002)$ & $2.773(0.003)$ & $0.811(0.001)$ & $2.967(0.000)$ \\
30 & $0.515(0.004)$ & $2.551(0.010)$ & $0.637(0.001)$ & $2.841(0.005)$ & $0.757(0.001)$ & $3.080(0.003)$ \\
40 & $0.477(0.003)$ & $2.573(0.016)$ & $0.590(0.002)$ & $2.876(0.015)$ & $0.712(0.003)$ & $3.181(0.012)$ \\
50 & $0.432(0.002)$ & $2.56(0.03)$ & $0.541(0.005)$ & $2.92(0.03)$ & $0.659(0.004)$ & $3.236(0.016)$ \\
60 & $0.380(0.002)$ & $2.51(0.02)$ & $0.486(0.002)$ & $2.915(0.018)$ & $0.602(0.003)$ & $3.282(0.016)$ \\
70 & $0.333(0.003)$ & $2.50(0.03)$ & $0.429(0.004)$ & $2.92(0.02)$ & $0.543(0.002)$ & $3.375(0.017)$ \\
80 & $0.283(0.003)$ & $2.53(0.03)$ & $0.377(0.002)$ & $3.07(0.03)$ & $0.478(0.005)$ & $3.56(0.05)$ \\
90 & $0.255(0.006)$ & $3.13(0.09)$ & $0.341(0.002)$ & $3.755(0.012)$ & $0.428(0.005)$ & $4.22(0.07)$ \\
100 & $0.330(0.004)$ & $6.38(0.06)$ & $0.391(0.002)$ & $6.61(0.04)$ & $0.461(0.002)$ & $6.79(0.03)$ \\
\hline
\end{tabular}


Table 4: Activation thermodynamics $(\mathrm{kJ} / \mathrm{mol})$ of hydrogen bond breaking in solutions of $\mathrm{MeOH}, \mathrm{EtOH}$ and $\mathrm{PrOH}$ as a function of concentration at $\mathrm{T}=298.15$.

Errors are given within parentheses, the error in $T \Delta \mathrm{S}$ is identical to that in $\Delta \mathrm{H}$.

\begin{tabular}{cccccccccc} 
Mass & \multicolumn{2}{c}{$\mathrm{MeOH}$} & \multicolumn{2}{c}{$\mathrm{EtOH}$} \\
$\%$ & $\Delta \mathrm{G}^{\ddagger}$ & $\Delta \mathrm{H}^{\ddagger}$ & $T \Delta \mathrm{S}^{\ddagger}$ & $\Delta \mathrm{G}^{\ddagger}$ & $\Delta \mathrm{H}^{\ddagger}$ & $T \Delta \mathrm{S}^{\ddagger}$ & $\Delta \mathrm{G}^{\ddagger}$ & $\Delta \mathrm{H}^{\ddagger}$ & $T \Delta \mathrm{S}^{\ddagger}$ \\
\hline 0 & $4.98(0.01)$ & $8.9(0.1)$ & 3.9 & $4.98(0.01)$ & $8.9(0.1)$ & 3.9 & $4.98(0.01)$ & $8.9(0.1)$ & 3.9 \\
10 & $5.24(0.00)$ & $9.8(0.1)$ & 4.5 & $5.30(0.00)$ & $10.3(0.2)$ & 5.0 & $5.25(0.00)$ & $10.4(0.1)$ & 5.2 \\
20 & $5.49(0.01)$ & $10.5(0.1)$ & 5.0 & $5.60(0.01)$ & $11.5(0.1)$ & 5.9 & $5.45(0.02)$ & $11.3(0.5)$ & 5.8 \\
30 & $5.71(0.01)$ & $10.9(0.2)$ & 5.2 & $5.88(0.06)$ & $12.6(0.3)$ & 6.8 & $5.65(0.01)$ & $11.8(0.5)$ & 6.1 \\
40 & $5.91(0.01)$ & $11.2(0.1)$ & 5.3 & $6.16(0.02)$ & $14.2(0.3)$ & 8.0 & $5.84(0.02)$ & $12.4(0.5)$ & 6.5 \\
50 & $6.09(0.02)$ & $11.3(0.2)$ & 5.2 & $6.40(0.05)$ & $14.4(1.2)$ & 8.0 & $6.05(0.05)$ & $13.1(0.5)$ & 7.1 \\
60 & $6.24(0.02)$ & $11.1(0.4)$ & 4.8 & $6.65(0.05)$ & $15.2(0.5)$ & 8.6 & $6.32(0.03)$ & $14.5(0.6)$ & 8.2 \\
70 & $6.34(0.05)$ & $9.8(0.2)$ & 3.4 & $6.90(0.04)$ & $15.2(0.5)$ & 8.3 & $6.63(0.06)$ & $15.6(0.7)$ & 8.9 \\
80 & $6.35(0.02)$ & $8.6(0.6)$ & 2.3 & $7.11(0.09)$ & $14.6(1.3)$ & 7.5 & $6.95(0.04)$ & $16.8(1.2)$ & 9.9 \\
90 & $6.32(0.02)$ & $7.1(0.1)$ & 0.7 & $7.16(0.04)$ & $11.9(1.4)$ & 4.7 & $7.19(0.04)$ & $16.7(2.0)$ & 9.5 \\
100 & $6.31(0.02)$ & $7.5(0.4)$ & 1.2 & $7.00(0.05)$ & $9.6(1.0)$ & 2.6 & $6.85(0.03)$ & $9.9(0.9)$ & 3.0 \\
\hline
\end{tabular}


Table 5: Equilibrium thermodynamics $(\mathrm{kJ} / \mathrm{mol})$ of hydrogen bond breaking in solutions of $\mathrm{MeOH}, \mathrm{EtOH}$ and $\mathrm{PrOH}$ as a function of concentration at $\mathrm{T}=298.15$.

Errors are given within parentheses, the error in $T \Delta \mathrm{S}$ is identical to that in $\Delta \mathrm{H}$.

\begin{tabular}{cccccccccc} 
Mass & \multicolumn{2}{c}{$\mathrm{MeOH}$} & \multicolumn{3}{c}{$\mathrm{EtOH}$} & \multicolumn{3}{c}{$\operatorname{PrOH}$} \\
$\%$ & $\Delta \mathrm{G}$ & $\Delta \mathrm{H}$ & $\mathrm{T} \Delta \mathrm{S}$ & $\Delta \mathrm{G}$ & $\Delta \mathrm{H}$ & $\mathrm{T} \Delta \mathrm{S}$ & $\Delta \mathrm{G}$ & $\Delta \mathrm{H}$ & $T \Delta \mathrm{S}$ \\
\hline 0 & $5.110(0.003)$ & $9.02(0.11)$ & 4.05 & $5.110(0.003)$ & $9.02(0.11)$ & 4.05 & $5.110(0.003)$ & $9.02(0.11)$ & 4.05 \\
10 & $5.217(0.004)$ & $9.66(0.06)$ & 4.60 & $5.212(0.004)$ & $9.87(0.06)$ & 4.82 & $5.140(0.005)$ & $9.86(0.08)$ & 4.89 \\
20 & $5.301(0.004)$ & $10.17(0.07)$ & 5.04 & $5.275(0.005)$ & $10.49(0.08)$ & 5.40 & $5.113(0.005)$ & $10.22(0.07)$ & 5.29 \\
30 & $5.366(0.005)$ & $10.50(0.07)$ & 5.31 & $5.306(0.005)$ & $10.94(0.09)$ & 5.83 & $5.049(0.006)$ & $10.35(0.09)$ & 5.49 \\
40 & $5.430(0.005)$ & $10.86(0.08)$ & 5.61 & $5.309(0.006)$ & $11.40(0.11)$ & 6.30 & $4.983(0.010)$ & $10.55(0.11)$ & 5.75 \\
50 & $5.487(0.007)$ & $11.19(0.09)$ & 5.90 & $5.289(0.007)$ & $11.58(0.12)$ & 6.50 & $4.916(0.008)$ & $10.90(0.14)$ & 6.19 \\
60 & $5.555(0.007)$ & $11.60(0.11)$ & 6.26 & $5.271(0.008)$ & $11.72(0.14)$ & 6.68 & $4.823(0.011)$ & $10.91(0.16)$ & 6.30 \\
70 & $5.629(0.007)$ & $11.91(0.12)$ & 6.50 & $5.258(0.015)$ & $11.93(0.15)$ & 6.89 & $4.725(0.013)$ & $11.2(0.2)$ & 6.7 \\
80 & $5.702(0.008)$ & $12.40(0.14)$ & 6.92 & $5.289(0.013)$ & $12.30(0.19)$ & 7.25 & $4.612(0.018)$ & $11.4(0.2)$ & 7.1 \\
90 & $5.729(0.011)$ & $12.54(0.17)$ & 7.05 & $5.417(0.015)$ & $13.2(0.3)$ & 8.1 & $4.57(0.02)$ & $12.7(0.3)$ & 8.4 \\
100 & $5.568(0.015)$ & $12.6(0.2)$ & 7.3 & $5.602(0.019)$ & $14.4(0.3)$ & 9.1 & $4.66(0.03)$ & $14.6(0.5)$ & 10.2 \\
\hline
\end{tabular}

